\title{
Successful Treatment of an Unresectable Inflammatory Myofibroblastic Tumor of the Frontal Bone Using a Cyclooxygenase-2 Inhibitor and Methotrexate
}

\author{
Fumiko Kusunoki-Nakamoto ${ }^{1}$, Takashi Matsukawa ${ }^{1}$, Masaki Tanaka ${ }^{1}$, Toji Miyagawa ${ }^{1}$, \\ Tomotaka Yamamoto ${ }^{1}$, Jun Shimizu ${ }^{1}$, Masako Ikemura ${ }^{2}$, Junji Shibahara ${ }^{2}$ and Shoji Tsuji ${ }^{1}$
}

\begin{abstract}
Inflammatory myofibroblastic tumor (IMT) is a disease characterized by tumorous lesions consisting of myofibroblastic spindle cells and inflammatory cells that occur primarily in the soft tissues and viscera of children and young adults. Total excision is the most effective therapy. Steroids have been used to treat unresectable lesions with some success. We herein report a case of IMT involving the frontal bone accompanied by pachymeningitis. The tumor was characterized by an aggressive clinical course that was refractory to prednisolone. Performing total excision seemed difficult. Celecoxib and methotrexate were effective treatments. Our experience suggests the efficacy of celecoxib and methotrexate as alternatives for treating unresectable IMT.
\end{abstract}

Key words: inflammatory myofibroblastic tumor, inflammatory pseudotumor, steroid therapy, pachymeningitis, COX-2 inhibitor, methotrexate

(Intern Med 52: 623-628, 2013)

(DOI: 10.2169/internalmedicine.52.8785)

\section{Introduction}

Inflammatory myofibroblastic tumor (IMT) is a rare disease characterized by tumorous lesions consisting of myofibroblastic spindle cells and inflammatory cells that occur primarily in the soft tissues and viscera of children and young adults. IMT can affect any part of the body, although it is most commonly seen in the lungs, mesentery and omentum and usually follows a benign clinical course. IMT of the head and neck is rare, accounting for fewer than 5\% of all extrapulmonary cases. The most common location is the orbit followed by the meninges, paranasal sinuses, infratemporal fossa and soft tissues. Our review of the literature revealed only 60 reported cases of IMT affecting the cranial bone (1-5). IMT has been described under many different names, including inflammatory pseudotumor (IPT) and plasma cell granuloma, highlighting the complexity and variable histological characteristics of these tumors. These lesions were once thought to be reactive in nature but have come to be considered true soft tissue neoplasms that can relapse, be multifocal and metastasize to distant sites.

Making a diagnosis of IMT requires a histopathological examination because the radiological and clinical findings are generally nonspecific. There is a consensus that total surgical excision is the most effective therapeutic option in cases in which the tumors are surgically accessible. Steroid therapy has been used with some success in patients with surgically unresectable lesions. Other medical treatment options, however, are limited, including radiotherapy and/or adjuvant chemotherapy and treatment with immunosuppressive and anti-inflammatory drugs. Methotrexate, cyclophosphamide, vinca alkaloids, cyclosporine and azathioprine have been used; however, the response is variable and often poor (6-9).

We herein present the case of a patient with IMT of the frontal bone whose lesion, despite showing a limited response to high-dose methylprednisolone therapy followed by

${ }^{1}$ Department of Neurology, Graduate School of Medicine, The University of Tokyo, Japan and ${ }^{2}$ Department of Pathology, Graduate School of Medicine, The University of Tokyo, Japan

Received for publication August 16, 2012; Accepted for publication November 26, 2012

Correspondence to Dr. Shoji Tsuji, tsuji@m.u-tokyo.ac.jp 

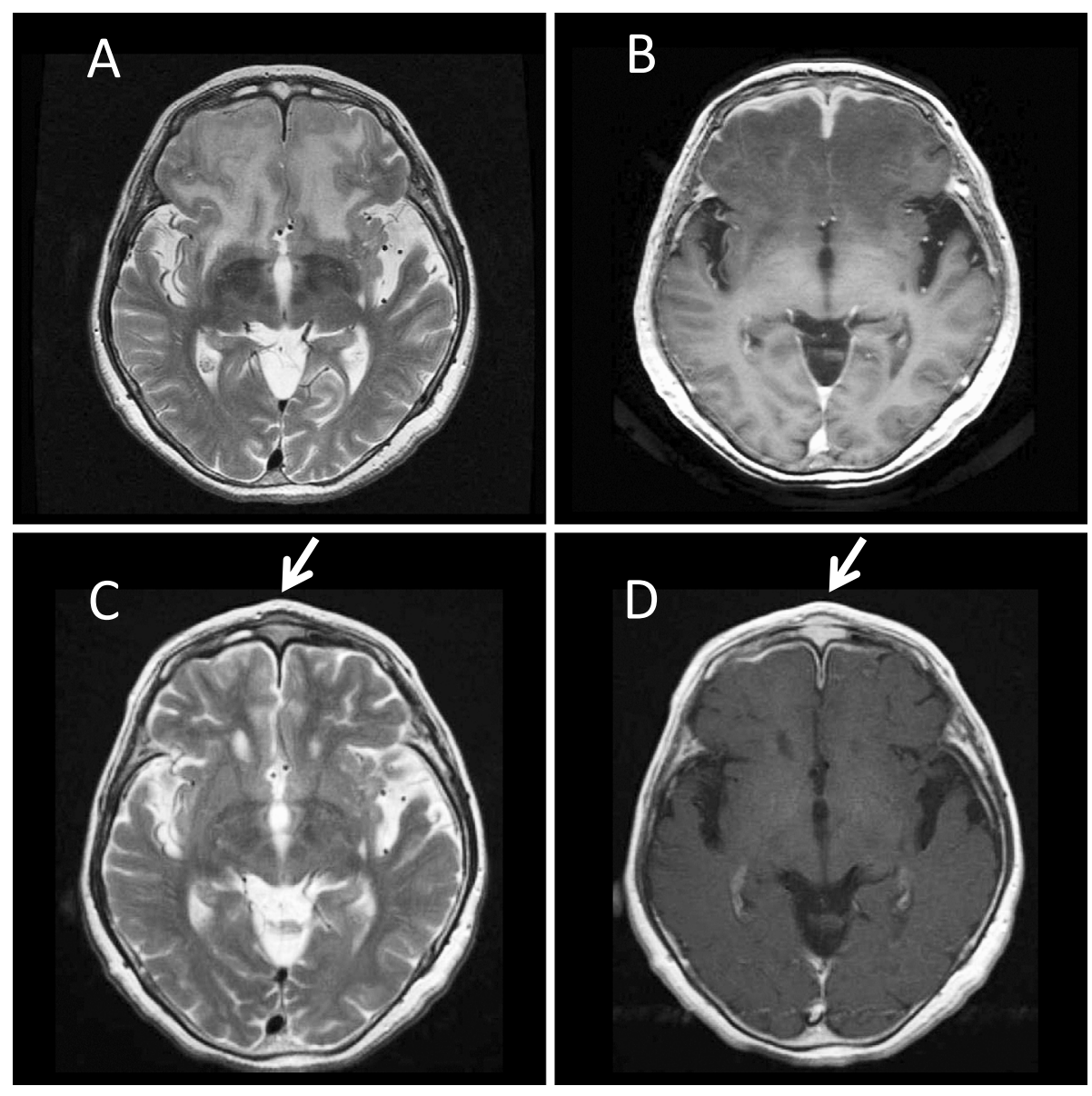

Figure 1. Brain MR images obtained on the first admission. (A, B) Brain MR images (A: axial T2weighted image, B: axial gadolinium-enhanced T1-weighted image) showing a thickened and enhanced dura mater and an enhanced lesion invading the parenchyma of the frontal lobe. Brain MR images obtained on the second admission (C: axial T2-weighted image, D: axial gadolinium-enhanced T1-weighted image). MR images showing a mass along the frontal bone. The mass appears enhanced on the images.

high-dose oral prednisolone (1 $\mathrm{mg} / \mathrm{kg}$ body weight/day), markedly shrank following the additional administration of a cyclooxygenase 2 (COX-2) inhibitor, celecoxib and methotrexate (MTX) therapy. To the best of our knowledge, this is the first report of a case of IMT that showed a limited response to steroid monotherapy alone but was treated successfully with a combination of steroids, MTX and a COX-2 inhibitor.

\section{Case Report}

A 64-year-old woman was admitted with progressively worsening vision in the right eye that had developed over the previous one month along with one episode of a tonic seizure. Four years before admission, she had undergone complete resection of an IMT of the right femur.

Magnetic resonance imaging (MRI) of the brain showed dural enhancement and thickening, and the frontal lobe was edematous (Fig. 1A, B). MRI also revealed a mass in the maxillary sinuses. A biopsy of the nasal cavity mucosa disclosed a nonspecific chronic inflammatory reaction. Special stains for bacteria, fungi and acid-fast organisms revealed negative findings. An immunohistochemical analysis of plasma cells and lymphocytes revealed the presence of both kappa- and lambda-type light chains, demonstrating the polyclonal nature of the plasma cells. Various examinations were conducted; however, no infectious or autoimmune diseases or hematological disorders were detected. The patient was diagnosed as having idiopathic hypertrophic pachymeningitis (IHP). Following the administration of methylprednisolone pulse therapy $(1,000 \mathrm{mg} / \mathrm{d}$, administered intravenously for three days), the patient's clinical symptoms and neuroradiological findings improved. She was kept on oral steroid therapy (40 $\mathrm{mg}$ of prednisolone per day) with gradual dose tapering.

The patient was admitted to the Department of Otolaryngology three years later for an evaluation of a nodule in her forehead that had been present for the past two months. She had no fever, fatigue or weight loss. A neurological examination revealed horizontal gaze nystagmus, hyperreflexia of the four limbs, dysesthesia of the bilateral toes, bilateral anosmia and conductive hearing loss in her left ear, which 


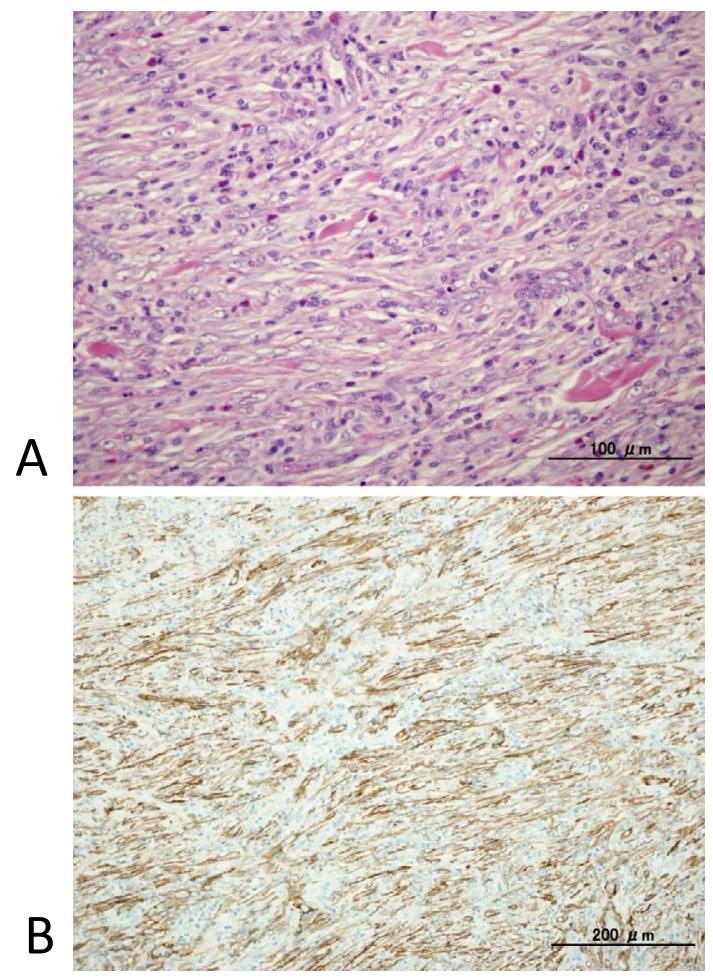

Figure 2. Histopathological findings of the frontal sinus biopsy specimens. (A) Frontal sinus biopsy specimens with Hematoxylin and Eosin staining showing many spindle cells with low proliferative activity and focal plasma cell infiltration indicative of IMT. (B) Immunohistochemical staining for smooth muscle actin showing that the majority of the spindle cells were positive for smooth muscle actin.

were all previously detected without additional neurological deficits. Tests for anti-nuclear antibodies, cytoplasmic antineutrophil cytoplasmic antibodies (C-ANCA), myeloperoxidase ANCA (P-ANCA), anti-Sjögren syndrome A (SS-A) antibodies, anti-SS-B antibodies, angiotensin-converting enzyme (ACE), beta-D glucan, soluble interleukin-2 receptor and immunoglobulin G4 were negative. MRI revealed an enhanced soft-tissue mass of the forehead with erosion of the frontal bone and dural thickening (Fig. 1C, D). A biopsy was performed, and a histopathological analysis of the obtained biopsy specimen revealed spindle cells without cellular atypism and marked infiltration of lymphocytes, plasma cells and neutrophils (Fig. 2A). Immunostaining demonstrated that the spindle cells were positive for $\alpha$-smooth muscle actin (Fig. 2B) and vimentin. On the basis of these findings, a histopathological diagnosis of IMT was made. The anaplastic lymphoma kinase (ALK) expression was negative, and the ratio of $\operatorname{IgG} 4+/ \mathrm{IgG}+$ plasma cells was not elevated. The dural thickening (typically seen in patients with IHP) seemed to be due to the lesions accompanying the IMT. Because performing complete resection was difficult and rapid growth of the tumor was observed, high-dose steroid therapy was chosen to treat the patient. Methylprednisolone pulse therapy was administered followed by oral prednisolone at a dose of $45 \mathrm{mg}$ daily. Seven weeks later, despite the fact that the patient was still on prednisolone, a computed tomography (CT) scan demonstrated that the tumor had further markedly increased in size (Fig. 3A-C). Two additional courses of methylprednisolone pulse therapy were administered, and the tumor growth appeared to stop. The third course was started followed by treatment with MTX (6 mg/week) and celecoxib $(200 \mathrm{mg} / \mathrm{d})$ in addition to oral prednisolone $(45 \mathrm{mg} / \mathrm{d})$. Two weeks later, marked shrinkage of the tumor was achieved (Fig. 3D). The patient was kept on oral prednisolone $(45 \mathrm{mg} / \mathrm{d})$ for 11 weeks, and the prednisolone dose was then gradually tapered down to $20 \mathrm{mg} / \mathrm{d}$ after four months. In the six months of follow-up after the patient's discharge, there has been no radiological evidence of any tumor progression or recurrence.

\section{Discussion}

In the present case, various examinations were conducted; however, no infectious or autoimmune diseases or hematological disorders were detected. On the patient's first admission, the tumor in the maxillary sinus and the dural thickening showed a marked improvement following the administration of methylprednisone pulse therapy. An immunohistochemical analysis of the biopsy specimen of the mass in the frontal bone, which developed three years later, showed spindle-shaped cells that were strongly positive for smooth muscle actin and vimentin. These findings favor a diagnosis of IMT (or inflammatory pseudotumor). Although the ALK expression was negative, this does not necessarily exclude a diagnosis of IMT because immunohistochemical positivity for ALK is observed in only approximately $50 \%$ of cases of IMT, more frequently in pediatric than adult patients.

It was difficult to consider the tumors of the paranasal sinuses to be distant metastatic lesions originating from the femur. Our patient showed no pathological evidence of malignancy. In addition, distant metastasis of IMT is rare, occurring in $<5 \%$ of cases, and metastatic disease is usually identified on presentation or within a year of diagnosis (10). However, the rapid dissemination to the paranasal sinuses and the frontal bone suggests the malignant nature of the disease. Our patient showed no pathological evidence of malignancy, although the clinical course was atypical for a benign tumor.

In our case, it is unclear whether the dural thickening was caused by IMT or HP. There are cases of HP resulting from IMT of the retropharynx or temporal bone (11), and some reports suggest that IMT and HP are part of a spectrum of disorders that have similar histopathologic findings (1). In such cases, it is unclear whether the dural thickening is due to direct spread of the tumor or inflammatory changes developing secondary to the tumor lesions because IMT occurring in the central nervous system is often dura-based.

It is difficult to determine the individual efficacies of the COX-2 inhibitor and MTX in our case because both drugs were administered simultaneously. There are case reports of IMT being successfully treated with nonsteroidal anti- 


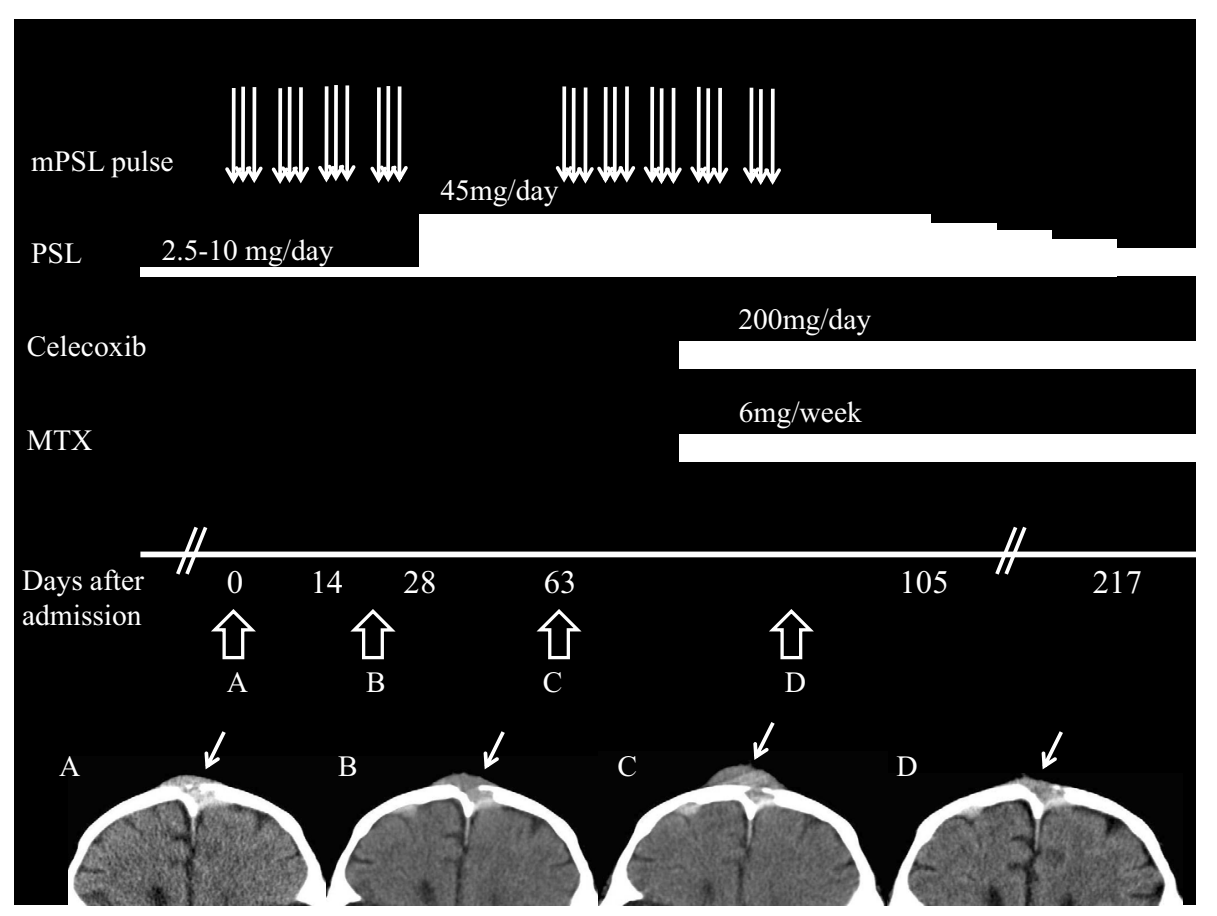

Figure 3. Clinical course and cranial CT images of the patient. (A) A cranial CT image obtained on the second admission showing a mass replacing the frontal bone. (B) A cranial CT image obtained 18 days after the initiation of methylprednisolone pulse therapy demonstrating that the mass remained the same and no reduction in size was achieved. (C) Although oral prednisolone was continued (45 mg/d), the CT image demonstrates progressive enlargement of the tumor. Methylprednisolone pulse therapy was readministered, and while the patient was still on steroid therapy, adjuvant therapy [methotrexate and celecoxib] was initiated. (D) A cranial CT image obtained two weeks after the initiation of adjuvant therapy showing marked shrinkage of the tumor.

inflammatory drugs (NSAIDs) and COX-2 inhibitors (Table) (11-19). Furthermore, we found several cases of IMT that were successfully treated with NSAIDs or a COX-2 inhibitor without the use of any surgery or any other medications.

The rationale for the use of NSAIDs in patients with IMT was demonstrated by Applebaum et al. (20), who convincingly presented evidence of the presence of mediators of angiogenesis, vascular endothelial growth factor (VEGF) and COX-2 that may play important roles in the growth of IMT. Niederberger et al. showed that COX-2 is expressed in activated endothelial cells and that COX-2 inhibition can directly suppress the growth of these cells, suggesting a possible role of COX-2 in endothelial regulation (21). Therefore, there are three possible mechanisms underlying the therapeutic role of COX-2 inhibitors in IMT: (1) an antiangiogenic effect occurring via VEGF downregulation, (2) suppression of COX-2-mediated endothelial proliferation, and (3) anticytokine suppression of the inflammatory process (20). In our patient, celecoxib and MTX markedly shrank the tumor, while steroid monotherapy showed partial effects of preventing tumor growth. Therefore, it is possible that the antiangiogenic actions of the COX-2 inhibitor were more effective for treating the IMT than the steroids and that celecoxib was responsible for the rather favorable response observed in our patient.
On the other hand, in our case, MTX was administered concomitantly with the COX-2 inhibitor. The role of MTX is, however, not clear. The tumor markedly decreased in size, as demonstrated on CT performed two weeks after the additional administration of MTX, although one or two months are generally needed until the peak efficacy of MTX is achieved. In addition, although various regimens, including those with MTX, that is MTX plus vincristine, MTX plus vinblastine and MTX plus cisplatin/adriamycin, have been described in the literature, there are no reports suggesting the efficacy of MTX monotherapy for IMT (6-9).

Some reports indicate that the combination of MTX with celecoxib enhances MTX-related effects (8); however, administering the two drugs at conventional clinical doses does not alter the pharmacokinetics of MTX because the concentration of serum MTX does not reach the inhibition constant (Ki) value. On the other hand, several authors have provided evidence that COX-2 inhibitors are considerably more effective when administered in combination with a secondary treatment regimen (22), which raises the possibility that COX-2 inhibitors can be more effective when administered in combination with MTX. In our case, therefore, the use of the COX-2 inhibitor alone or administered in combination with MTX was presumably effective in decreasing the size of the tumor.

IMT is a rare and difficult condition to manage. The opti- 
Intern Med 52: 623-628, 2013 DOI: 10.2169/internalmedicine.52.8785

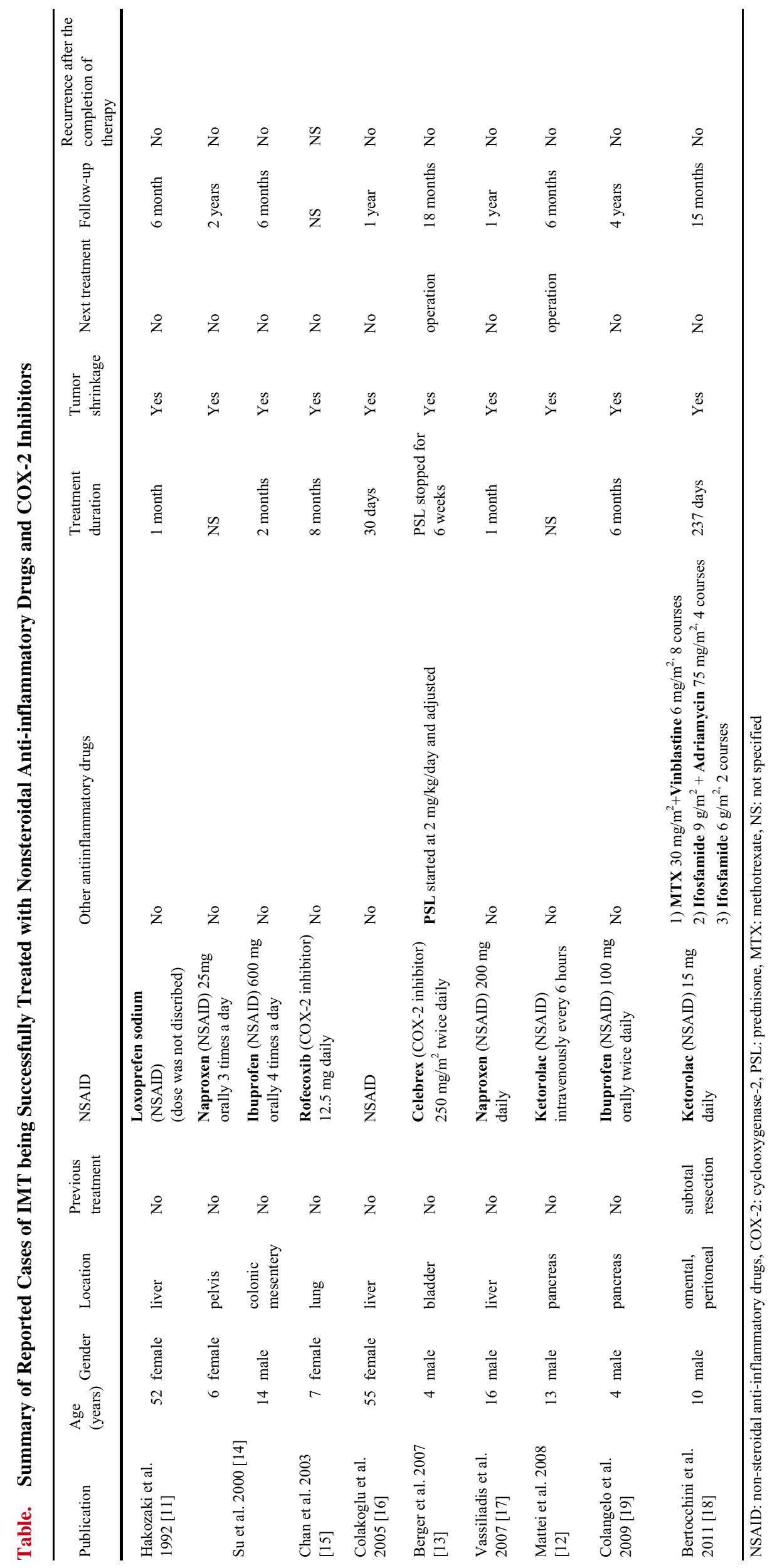


mal management of locally aggressive and metastatic forms is still under debate and should be decided on a case-bycase basis. Our experience suggests the clinical advantage of using a COX-2 inhibitor as part of the adjuvant therapy.

The authors state that they have no Conflict of Interest (COI).

\section{References}

1. McKinney AM, Short J, Lucato L, SantaCruz K, Mckinney Z, Kim Y. Inflammatory myofibroblastic tumor of the orbit with associated enhancement of the meninges and multiple cranial nerves. Am J Neuroradiol 27: 2217-2220, 2006.

2. Ajibade DV, Tanaka IK, Paghdal KV, Mirani N, Lee HJ, Jyung RW. Inflammatory pseudotumor (plasma cell granuloma) of the temporal bone. Ear Nose Throat J 89: E1-E13, 2010.

3. Kaye AH, Hahn JF, Craciun A, Hanson M, Berlin AJ, Tubbs RR. Intracranial extension of inflammatory pseudotumor of the orbit. J Neurosurg 60: 625-629, 1984.

4. Schönermark MP, Issing P, Stöver T, Ruh S, Lenarz T. Fibroinflammatory pseudotumor of the temporal bone. Skull Base Surg 8 : 45-50, 1998.

5. Gale N, Zidar N, Podboy J, Valašvek M, Luzar B. Inflammatory myofibroblastic tumour of paranasal sinuses with fatal outcome: reactive lesion or tumour? J Clin Pathol 56: 715-717, 2003.

6. Trojan A, Stallmach T, Kollias S, Pestalozzi BC. Inflammatory myofibroblastic tumor with CNS involvement. Onkologie 24: 368372, 2001.

7. Sacco O, Gambini C, Gardella C, et al. "Atypical steroid response" in a pulmonary inflammatory myofibroblastic tumor. Pediatr Pulmonol 45: 721-726, 2010.

8. Dishop MK, Warner BW, Dehner LP, et al. Successful treatment of inflammatory myofibroblastic tumor with malignant transformation by surgical resection and chemotherapy. J Pediatr Hematol Oncol 25: 153-158, 2003.

9. Favini F, Resti AG, Collini P, et al. Inflammatory myofibroblastic tumor of the conjunctiva: response to chemotherapy with low-dose methotrexate and vinorelbine. Pediatr Blood Cancer 54: 483-485, 2010.

10. Gleason BC, Hornick JL. Inflammatory myofibroblastic tumours: where are we now? J Clin Pathol 61: 428-437, 2008.

11. Hakozaki Y, Katou M, Nakagawa K, Shirahama T, Matsumoto T. Improvement of inflammatory agent therapy. Am J Gastroenterol 88: 1121-1122, 1995.

12. Mattei P, Barnaby K. Rapid regression of duodenal inflammatory myofibroblastic tumor after intravenous ketorolac: case report and review of the literature. J Pediatr Surg 43: 1196-1199, 2008.

13. Berger A, Kim C, Hagstrom N, Ferrer F. Successful preoperative treatment of pediatric bladder inflammatory myofibroblastic tumor with anti-inflammatory therapy. Urology 70: 372.e13-372.e15, 2007.

14. Su W, Ko A, O'Connell T, Applebaum H. Treatment of pseudotumors with nonsteroidal anti-inflammatory drugs. J Pediatr Surg 35: 1635-1637, 2000.

15. Chan PW, Omar KZ, Ramanujam TM. Successful treatment of unresectable inflammatory pseudotumor of the lung with COX-2 inhibitor. Pediatr Pulmonol 36: 167-169, 2003.

16. Colakoglu O, Unsal B, Haciyanli M, et al. A successfully managed inflammatory pseudotumour of liver without surgery: report of a case. Acta Gastroenterol Belg 68: 382-384, 2005.

17. Vassiliadis $\mathrm{T}$, Vouguiouklis $\mathrm{N}$, Patsiaoura $\mathrm{K}$, et al. Inflammatory pseudotumor of the liver successfully treated with nonsteroidal anti-inflammatory drugs: a challenge diagnosis for one not so rare entity. Eur J Gastroenterol Hepatol 19: 1016-1020, 2007.

18. Bertocchini A, Lo Zupone $C$, Callea F, et al. Unresectable multifocal omental and peritoneal inflammatory myofibroblastic tumor in a child: revisiting the role of adjuvant therapy. J Pediatr Surg 46: e17-e21, 2011

19. Colangelo M, Lisi G, Chiesa PL. Pancreatic inflammatory myofibroblastic tumor (IMT). J Pediatr Surg 45: 1074-1075, 2010.

20. Applebaum H, Kieran MW, Cripe TP, et al. The rationale for nonsteroidal anti-inflammatory drug therapy for inflammatory myofibroblastic tumors: a Children's Oncology Group study. J Pediatr Surg 40: 999-1003, 2005.

21. Niederberger E, Manderscheid C, Grösch S, Schmidt H, Ehnert C, Geisslinger G. Effects of the selective COX-2 inhibitors celecoxib and rofecozib on human vascular cells. Biochem Pharmacol 68: 341-350, 2004.

22. Klenke FM, Abdollahi A, Bischof M, et al. Celecoxib enhances radiation response of secondary bone tumors of a human nonsmall cell lung cancer via antiangiogenesis in vivo. Strahlenther Onkol 187: 45-51, 2011.

(C) 2013 The Japanese Society of Internal Medicine http://www.naika.or.jp/imonline/index.html 